\title{
It's Not Just If You See It, It's How You Process It: Conceptual and Perceptual Fluency Effects for Brand Names
}

\author{
By Temple Northup*
}

\begin{abstract}
There has been a consistent body of research that has shown that increases in accessibility of an item within memory, often referred to has processing fluency, causes there to be more positive affect toward that item. This relationship has particular importance for advertising and consumer behavior research as one of the outcomes of an advertising campaign is an increase in brand salience. Although there has been interest in investigating the relationship between fluency and attitudes in relation to brands, little research has attempted to distinguish between the two different types of fluency that exist: perceptual and conceptual. Over three studies, this research investigated the extent to which perceptual versus conceptual fluency impacted attitudes toward known brands, with results suggesting that it is conceptual fluency that makes a significant contribution.
\end{abstract}

Keywords: advertising, affective judgment, implicit memory, processing fluency.

\section{Introducing}

An underlying assertion in research examining "fluency" effects (e.g., the mere exposure effect, Zajonc, 1968) is that exposure to a stimulus item causes it to become more accessible in memory. This increase in accessibility enhances the ease with which it can be identified or recalled, which is known as processing fluency. Past research has found that processing fluency is affectively positive (Hansen \& Wanke, 2009) and can be conceptually or perceptually based (Tulving \& Schacter, 1990). Conceptual fluency relates to the ease with which an item can come to mind when thought about in a semantic or meaningful manner, whereas perceptual fluency reflects how easily and quickly the surface features of an item can be recalled. For example, within the domain of consumer research, if someone is exposed to an advertisement for the gum Orbit, conceptual fluency could be reflected in how quickly and readily that item (Orbit) comes to mind when the category of gum is presented, whereas perceptual fluency could be reflected in how quickly an image of the product Orbit could be identified (e.g., if flashed momentarily on a screen).

Butler and Berry (2004) argue that implicit memory is what drives the increase in perceptual or conceptual fluency. Implicit memory is "revealed when previous experiences facilitate performance on a task that does not require conscious or intentional recollection of those experiences" (Schacter, 1987, p. 501). In the example above, the "previous experience" would be exposure to Orbit and the performance facilitation would be the top of the mind awareness or ability to recognize the brand. The purpose of this research is to

\footnotetext{
${ }^{*}$ Director \& Associate Professor, Jack J. Valenti School of Communication, University of Houston USA.
} 
explore the relationship between fluency and attitudes by using implicit memory procedures to gauge and estimate how accessible certain brands are in memory and see what relationship, if any, emerges between that accessibility and affective evaluations.

\section{Literature Review}

In the literature on implicit memory, researchers differentiate between perceptual and conceptual forms of implicit memory. Perceptual forms of memory tend to relate to a shallow form of encoding whereas conceptual forms relate to a deeper processing. Tests of perceptual implicit memory feature fragmentary or rapidly-presented cues that the participant attempts to identify. In contrast, conceptual implicit tests present participants with cues that are meaningfully related to the to-be-retrieved material. An example is the category production task in which category names are used to elicit examples (see Mulligan, 2004; Roediger \& McDermott, 1993, for reviews).

There has been a steady increase in the use of both perceptual and conceptual implicit memory measures in advertising research (e.g., Choi, Lee, \& Li, 2013; Coates, Butler, \& Berry, 2004, 2006; Finlay, Marmurek, \& Morton, 2005; Hervet, Guerard, Tremblay, \& Chtourou, 2011; Krishnan \& Shapiro, 1996; Lee, 2002; Vandeberg, Murre, Voorveld, \& Smit, 2015; Yoo 2007, 2008, 2010). For instance, Yang, Roskos-Ewoldsen, Dinu, \& Arpan, 2006; Yang \& Roskos-Ewoldsen, 2007), who investigated perceptual implicit memory for product and brand placements within movies and video games, found similar levels of perceptual implicit memory performance for products in movies and video games regardless of whether they were heavily featured in the storyline (or game). In contrast, Northup and Mulligan (2013a, 2013b) used a category-exemplar production task-a conceptual implicit memory measure - to test for priming after participants were presented with brand names. Their results demonstrated that conceptual priming could be found for brands presented as words (just the brand name), images (with the brand name clearly identifiable in the image), or ads embedded within a website.

Together, these studies suggest exposure to a brand name-whether through advertisements, product placements, or any number of other mechanisms - can increase accessibility of that brand in memory. This accessibility can be measured via perceptual or conceptual measures, which reflect different types of processing fluency. However, to date, little extant research has tried to connect the extent to which increased brand fluency as measured by implicit memory procedures correlate with positive affect.

The research that does exist suggests increases in accessibility may lead to increased positive affect. Indeed, the perceptual fluency/attributional model, developed by Bornstein and D'Agonstino (1992, 1994), posits that repeated exposure to a stimulus results in increased fluency for that item. That fluency translates into an increase of positive affect. Furthermore, when people experience that increase in fluency, they do not attribute the fluency to the prior 
presentation of the stimulus; rather, they generate an alternative but feasible explanation for the feeling of familiarity. In one of the few examples in which conceptual and perceptual fluency effects were tested together, advertisements received positive evaluations whether the ads were read or viewed in either a perceptual or a conceptual context (Lee \& Labroo, 2004). In other words, conceptual and perceptual fluency operated similarly.

Bornstein and D'Agonstino $(1992,1994)$ articulate the importance of awareness as key to understanding the effects of this increased affectspecifically; an awareness of the prior presentation can dampen or extinguish the change. For instance, when stimuli are presented subliminally, there is generally a robust increase in positive affect toward those stimuli. In contrast, for items presented supraliminally, there still may be an increase in positive affect, but it is typically not as pronounced. According to Bornstein and D'Agonstino, an awareness of the connection between presentation and fluency explains this reduction.

Within the implicit memory research, awareness is one of the most important aspects to be taken into account and controlled for as a lack of awareness is critical for a measure to be truly implicit. In the typical implicit memory procedure, participants are exposed to stimulus materials and then later complete the implicit memory measure. If the participant is aware during the memory measure that the responses are related to the earlier exposure, the implicit measure can no longer be considered implicit as explicit retrieval strategies may be used. To counter these effects, participants are typically either warned of the possibility that they may notice responses that are similar to earlier presentations and to ignore that (e.g., Parker \& Dagnall, 2009) or participants complete an awareness questionnaire after completion of the memory measure that helps to identify those who were using explicit retrieval strategies (e.g., Northup \& Mulligan, 2013b).

The purpose of this research, then, is to build on this previous research in two important ways. First, to specifically investigate the extent to which implicit conceptual and perceptual memory performance is a predictor of attitudes. In their model, Bornstein and D'Agonstino (1992, 1994) focused on perceptual fluency and therefore did not differentiate between conceptual and perceptual processing and how that distinction could lead to different relationships between fluency and affect. Second, the vast majority of applied research that has looked at effects related to processing fluency has relied on the use of brand names that are fictitious or not well known. This research aims to build on the previous literature by using known brands to examine any effects that may be present.

Based on this literature, the following two primary hypotheses are presented:

$\mathrm{H}_{1}$ : Perceptual implicit memory performance will positively correlate with attitudes toward brands in the advertisements.

$\mathrm{H}_{2}$ : Conceptual implicit memory performance will positively correlate with attitudes toward brands in the advertisements. 
In order to test these hypotheses, three studies were conducted. The first two were designed to investigate the relationship between conceptual and perceptual fluency for both brand names as well as brand logos. The third study builds on the first two studies by adding a control (non-exposure) group to gain a deeper understanding of the relationship between fluency, exposure, and attitudes.

\section{Study 1}

The purpose of the first study was to explore what relationship, if any, exists between perceptual or conceptual fluency and affect. One hundred ninety-eight students from a large, public university in the Southwestern United States (mean age $=20.32,112$ women) participated in a 2 condition (conceptual or perceptual implicit memory measure) between-subject experiment in which they, after being exposed to brand names presented within advertisements, performed either a conceptual or perceptual implicit memory test. They then participated in a forced-choice measure that included the earlier brands from the advertisements to gauge their attitudes toward those brands.

\section{Procedure}

At designated times, participants entered a computer laboratory. The first task the participants had to complete was to rate how familiar or unfamiliar they were with 21 brands, a task that previous research has shown will induce sufficient processing to elicit priming results on both conceptual and perceptual measures (Northup \& Mulligan, 2013a). Each brand name was presented within the context of an image (see Figure 1 for examples).

Figure 1. Example of Brand Names Presented Within Images
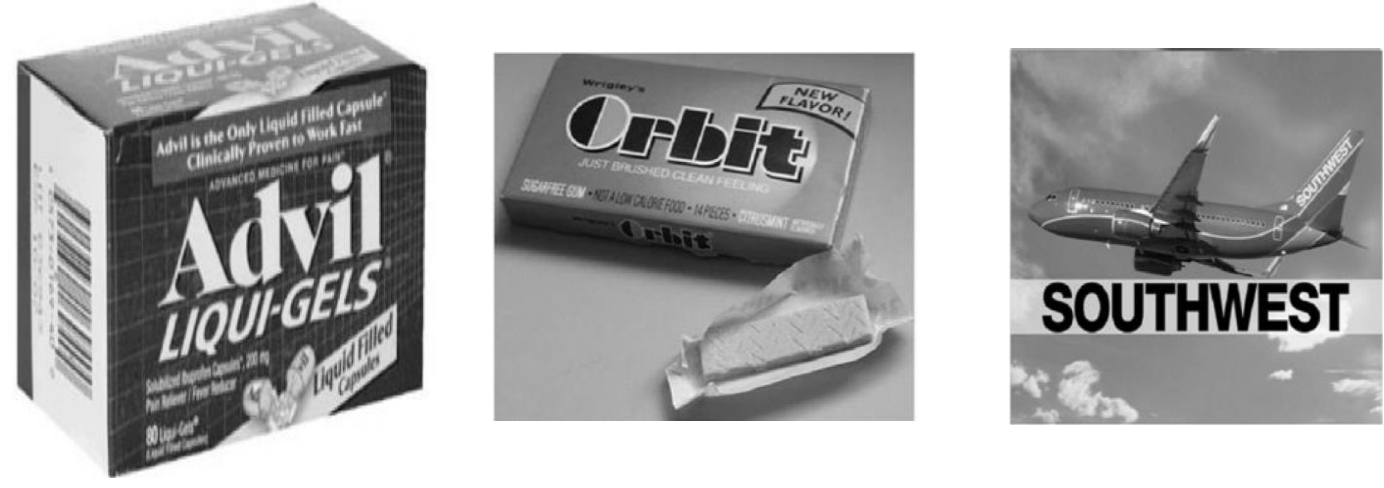

Importantly, 15 of the brands were the target brands with an additional six being fillers serving as recency and primacy buffers. After completing a 10-minute distraction task, participants were presented with either a conceptual or perceptual implicit memory test. For the conceptual test, participants were asked to generate the "first brand that comes to mind" for a number of categories (e.g., name a brand of gum). For the perceptual test, they were asked to complete a word fragment 
from a well-known brand name (e.g., O_b_t for Orbit). For either memory measure, the target categories or word fragments were preceded by 20 unrelated brand categories or brands so that participants became acclimated to the task. Furthermore, the target tasks were alternated with unrelated brands so that no two of the target brands were ever back-to-back. After completing the memory measure, the participants performed a forced-choice task where they had to choose between two brands within a similar category (e.g., choose between the following two shoe brands: Nike or New Balance), one of which related to the brands previously seen. The implicit memory performance serves as the independent variable while the performance on the forced-choice task serves as the dependent variable.

Of note, the procedure closely followed Parker and Dagnall (2009) to control for explicit contamination and keep the implicit memory measure implicit (i.e., non-intentional). To do this, participants were explicitly warned both when they were performing the memory measure and the forced-choice task that the brands they generate (or choose between) may be related to the earlier brands they evaluated and to not allow that to influence their results as the tasks were unrelated. Nevertheless, after the procedure, questions were asked as to whether, despite the warning, participants felt that they were using explicit retrieval strategies. Those participants who indicated they were using explicit strategies were removed from the main analysis. Worth noting, the number who indicated using explicit strategies in this and the subsequent study was small $(<10)$ and so results comparing the groups were not possible.

\section{Results and Discussion}

In line with previous research, to calculate the independent memory measures, responses for each target task (i.e., each brand generation or word fragment) were coded as either matching the earlier seen brand $(=1)$ or not $(=0)$. The responses to the 15 target brands were then summed and average to represent a proportion of brands generated during the implicit memory measure (conceptual $M=.20, S D=.13$; perceptual $M=.21, S D=.15$ ). A similar procedure was used for the dependent variable, with the key difference being that the brands were either chosen $(=1)$ or not chosen $(=0)$ in the forced-choice task $(M=.50, S D=.12)$.

To explore the relationship between fluency and affect, correlations were performed between conceptual and perceptual implicit memory performance and forced-choice performance. Results suggest there was a significant and positive correlation between conceptual memory performance and forced-choice, $r=.22$, $p=.04$; however, there was no significant relationship between perceptual memory performance and forced-choice, $r=.17, p=.11$, although the results did approach significance and were only slightly different than the conceptual correlation. Nevertheless, initial evidence from this first study does not support H1 as there was no relationship between perceptual fluency and affect, whereas $\mathrm{H} 2$ is supported as conceptual fluency and affect were related. This suggests that increased conceptual accessibility of brand names predicted the brands being 
chosen when given brand-pairs, whereas perceptual accessibility did not. This would mean that conceptual-based fluency is more strongly related to positive affect, at least when choosing between known brands.

\section{Study 2}

Study 2 closely mimicked Study 1 in procedure. The purpose was to first replicate the results of the first experiment and then change the design slightly, this time using logos instead of images of products. This was to determine whether the logo alone-that is, without any specific mention of the brand name and without the brand name in written text-is sufficient to increase conceptual or perceptual fluency, which in turn could increase positive affect.

\section{Procedure}

One hundred seventy-nine students from a large, public university in the Southwestern United States completed the procedure (mean age $=21.70$, $S D=3.10,118$ women). Participants took part in a similar procedure as the first study with the following change: instead of seeing the brand names for each brand, participants instead rated the logos associated with the brands. These logos did not include any text (see Figure 2 for examples).

Figure 2. Example of Logos
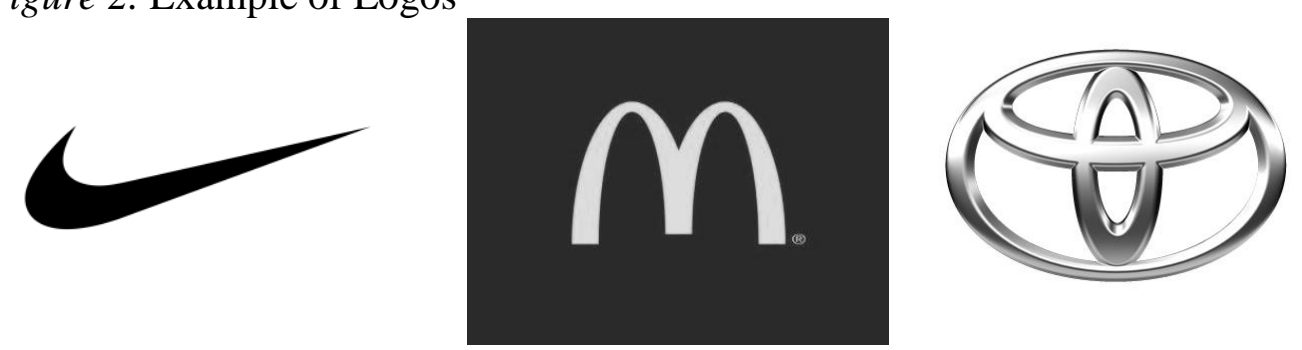

Participants rated the logo on familiarity with the brand name, which should again induce conceptual processing sufficient to activate increases in both conceptual and perceptual fluency. Beyond this change, the procedure was entirely identical to the first study.

\section{Results and Discussion}

Similar analyses were run as in the first study with correlations being performed between conceptual and perceptual implicit memory performance and forced-choice performance. Results suggest there was a significant and positive correlation between conceptual memory performance and forcedchoice, $r=.31, p<.001$; however, there was no significant relationship between perceptual memory performance and forced-choice, $r=.16, p=.13$, although the results again did begin to approach significance. As with Study 1, these results 
therefore do not support $\mathrm{H} 1$ as perceptual fluency did not correlate with affect, whereas $\mathrm{H} 2$ was supported as conceptual fluency and affect were related.

Although comparisons between correlational coefficients from different studies are difficult to do, it is worth noting that the correlation between conceptual fluency and positive affect was a little higher (that is, stronger) in the second study as compared to the first. This is interesting because the second study used logos that did not include the text name of the brand. One possible explanation of why there was a stronger correlation is that evaluating a brand logo alone causes the participants to first generate the brand name itself (that is, to come up with the name of the brand that is associated with the logo), and then to evaluate how familiar the brand is. If that is the case, it is possible that there is a deeper processing that occurs, which could increase the fluency and the subsequent increase in positive affect. Although this is merely one possible explanation, it is interesting to that the logo appears to have produced stronger results.

\section{Study 3}

Although the first two studies were compelling, a third study was run to build upon the results in a few important ways. First, this study would use an attitude instrument instead of a forced-choice measure. By changing the type of dependent variable, a more complete picture can begin to emerge in terms of the importance of different types of fluency. Second, this procedure was altered to attempt to more thoroughly isolate the effects of awareness on the participants, thereby making the implicit measures more "implicit." Finally, a control group was added to explore the extent to which the exposure to the brands was driving the effects obtained in the first two studies, as compared to a baseline level of fluency that might exist already.

\section{Procedure}

Two hundred eighty students from a large, public university in the Southwestern United States participated (mean age $=20.70,156$ women) in a similar procedure as the previous studies with the following changes. First, instead of rating brand names, participants viewed screen shots from news websites that included what was presented as an advertisement. Because previous research (Northup \& Mulligan, 2013b) has demonstrated that clicking an ad is sufficient to induce conceptual and perceptual processing, there were no instructions presented to the participants outside of clicking on the advertisement. In fact, the first part of the study was presented as a website usability task for online news websites with the participants going through a series of tasks for each website (e.g., click on the first thing you see; click on the link to the weather report; and, importantly, click on the advertisement). This was done to help mask the true purpose of the task. See Figure 3 for sample screen shots. 
Figure 3. Examples of News Website Screenshots with Created Advertisements

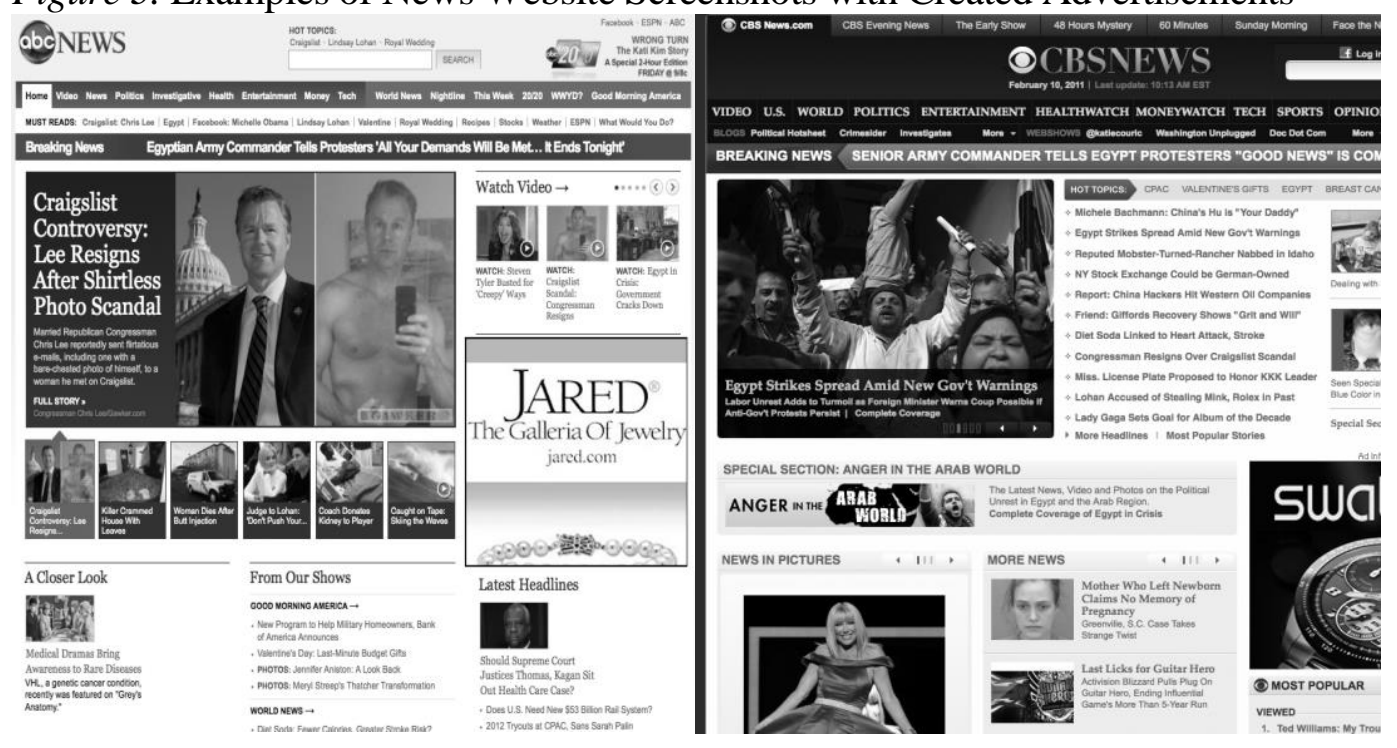

After participants finished a filler task, they proceeded to either the conceptual or perceptual implicit memory measure. There were also two "control" groups (one for conceptual fluency, one for perceptual fluency) who did not view the websites but began the procedure at this point. All participants then completed an attitude measurement for each of the brands presented within the websites. To do this, participants rated on a 7-point semantic differential scale their attitudes toward each brand on four dimensions: likedislike, good-bad, positive-negative, and favorable-unfavorable. Past research has suggested these four dimensions are valid measures of attitudes toward brands (MacKenzie, Lutz \& Belch, 1986). The four items that related to each participant's overall attitude toward each brand were averaged to create a mean attitude toward the brand. Each individual attitude measure was reliable (all alphas above .90). The means of all 15 attitudes toward the brands were then averaged across all brands to create a single, overall mean attitude variable to serve as the dependent variable.

Finally, participants who had viewed the websites completed an awareness questionnaire that included five questions to identify participants who may have "caught on" to the purpose of the experiment. This is another technique used to assess awareness in implicit memory research (Bowers \& Schacter, 1990). Following all the measures, participants are asked a series of increasingly specific questions about the purpose of the study. This test has been shown to be, if anything, conservative in its assessment of test aware versus unaware (Barnhardt \& Geraci, 2008). Those participants who indicated they were unaware of any connections were put into the test unaware group $(=0)$. All others were put into the test aware group $(=1)$. The advantage of this procedure, compared to the previous studies, is that it allows for the isolation of participants who were truly using implicit memory to guide their responses. 


\section{Results and Discussion}

The relationship between perceptual and conceptual fluency was investigated again using a bivariate correlation between memory performance (conceptual or perceptual) and attitudes. Because awareness has proven to be an important consideration, all analyses were conducted separately on the test aware and unaware groups.

Considering first perceptual fluency, regardless of whether the participants were test unaware, aware, or in the control group, none demonstrated a significant correlation between fluency and attitudes ( $r s$ of $.11, .20$, and .18 respectively, $p>.05$ ). These results replicate the first two studies and suggest that when making brand evaluations, perceptual fluency does not appear to have a strong relationship with attitudes. H1 was therefore not supported.

Considering conceptual fluency, those who were test unaware demonstrated a moderately strong correlation between implicit memory performance and attitudes, $r=.59, p<.001$. In contrast, those who were test aware did not show any relationship, $r=.05, p>.05$, nor did the control group, $r=.07, p>.05$. This also replicates the previous studies and suggests that conceptual fluency does correlate with positive affect-provided individuals are unaware of the source of their fluency. $\mathrm{H} 2$ is therefore supported. ${ }^{1}$

To further understand the relationship among exposure, fluency, and affect, steps were taken to assess whether exposure via website ads moderated the relationship between conceptual fluency and attitudes. In order to test this, a hierarchical regression was performed to predict attitudes toward the brands, with implicit memory performance as well as exposure $(0=$ no exposure, $1=$ exposure) entered in Step 1. The two-way interaction between exposure and memory performance was entered into Step 2. A significant two-way interaction would indicate a moderating relationship.

According to the results of Step 1, both exposure $(B=.42, S E B=.15, p<.01)$ and implicit memory performance $(B=2.20, S E B=.56, p<.001)$ predicted overall attitude performance, $R^{2}=.27, F(2,46)=8.56, p<.01$. The addition of the two-way interaction $(B=2.75, S E B=1.18, p<.05)$ in Step 2 yielded a significant improvement, $\Delta \mathrm{R}^{2}=.08, \Delta \mathrm{F}(1,45)=5.45, \mathrm{p}<.05$. Next, simple slope analyses were performed to examine the significant memory $\mathrm{X}$ exposure interaction. Simple slope analysis was conducted to isolate the contribution of exposure in overall attitudes, depending on the level of implicit memory performance. The slope of the resulting lines is larger for the "ad exposure" condition $(B=2.98)$ than the "no ad exposure" condition $(B=.23)$. According to subsequent one-tailed $t$-tests (Aiken $\&$ West, 1991, p. 17), the ad exposure slope was significantly different from zero $(t(45)=2.86, p<.05)$, whereas the no exposure slope was not $(t(45)=1.72, p>.05)$.

\footnotetext{
${ }^{1}$ Although not directly related to this research, it is worth pointing out that significant priming did occur - that is, those exposed to the advertisements generated the products more frequently than the control groups (all p values less than .01). For the conceptual tests, the memory performance after exposure $(M=.38, S D=.14)$ was higher than control $(M=.18, S D=.10)$. A similar pattern was observed for the perceptual performance after exposure $(M=.28, S D=.15)$ compared to perceptual control $(M=.17, S D=.11)$.
} 
These results suggest that exposure to the ad does indeed act as a moderator enhancing the effects of implicit conceptual fluency.

Taken together, the results of Study 3 generally give further support to the idea that conceptual rather than perceptual fluency has the most influence on affect at least with known brands. That is, exposure to brand through website advertisements increased conceptual fluency and triggered more positive evaluations - provided the participants were unaware of the source of that fluency.

\section{Conclusion}

Bornstein and D'Agostino's (1992, 1994) perceptual fluency/attributional model suggests that fluency is affectively positive-that is, increases in accessibility should translate to increases in positive affect. The purpose of this research was to examine the extent to which perceptual and conceptual fluency each predict feelings about a brand-measured in Studies 1 and 2 through a forced-choice instrument and in Study 3 by an attitude measurement. Although their original model was framed in terms of perceptual fluency, there was no reason to assume that conceptual fluency would act differently (Lee \& Labroo, 2004). However, results from these studies suggest that conceptual fluency is driving affect much more than perceptual fluency, which did not make a significant contribution in any of the studies. This implies that the type of memory accessibility matters when interested in brand attitudes-in other words, merely having a brand accessible is not the same as having that information accessible in a conceptual or meaningful manner.

One explanation for conceptual rather than perceptual fluency being more important is that this research used actual, known brands. Most previous research studies investigating fluency effects have used fictitious brand names or abstract items such as Chinese symbols (Zajonc, 1968). Under conditions when participants were unfamiliar with the attitude object, having it be merely accessible may be sufficient to create positive affect. However, when it comes to fluency and attitudes toward familiar objects, these results suggest it is conceptual rather than perceptual fluency that is essential. It is possible that small increases in perceptual fluency are simply not sufficient to cause any immediate or measurable changes in attitudes.

Also worth noting is that in Study 3, when differences based on awareness could be assessed, those classified as unaware showed a relationship between conceptual fluency and affect whereas those who were aware did not. This conforms well to Bornstein and D'Agostino's $(1992,1994)$ model as they specifically recognized that individuals needed to be unaware of the source of their fluency in order to show increased positive affect. Although much of their research compared those who were subliminally primed (and therefore unaware) to those who were explicitly shown stimuli, a parallel could be made here as those who did not realize that the brands they were generating or rating were the same they had seen earlier were presumably equally unaware of the source of their feelings. 
In fact, these results appear to fit well with Jacoby and Kelley's (1987) conceptualization of memory being capable of being used as an object or as a tool. When used as a tool, the focus is on the present task at hand and memory works to unconsciously influence behavior. When used as an object, memory is used as something to be searched and used in a much more effortful and intentional way. In the present research, those who treated memory as a tool focused on the attitude questions only and their memory - as measured through the increased conceptual fluency - guided their judgments. On the other hand, those who were aware of the connection between the tasks used memory as an object to be searched, helping them to understand the source of their conceptual fluency, which in turn helped to discount any increase in positive affect.

Similarly, these results suggest a type of sleeper effect may have occurred. Although time is typically seen as an important element of the sleeper effect, causing individuals to remember a message while forgetting the source of the message, Jacoby, Kelley, Brown, and Jasechko (1989) argued that it was not that time caused participants to forget the source; rather, time caused participants to not think back to the source of the message and instead use memory as a tool to influence attitudes. In other words, it is not that individuals forget the source, it is that individuals are cognitive misers who do not want to exert the energy it requires to remember the source. In the context of this study, then, it is possible that participants could have remembered that they had seen the brands before but did not try to recall them, which therefore means that they did not connect the source of their positive feelings with that previous exposure.

The results of Study 3 also suggest that advertisement exposure acts as a moderator between conceptual fluency and attitudes. Without exposure to the brand names, there were no differences in attitudes based on fluency; however, once exposure was taken into account, a relationship developed. In this way, it can be conceptually explained that the presence or absence of the advertisement is moderating the relationship between conceptual fluency and attitudes. On its face, this is of note because the baseline (control group's) fluency alone did not predict positive affect; rather, it was only once the advertisement exposure occurred that there was an increase in positive affect associated with the fluency.

Moving forward, the results of this research suggest that it is important not just to measure memory accessibility, but rather the type of memory accessibility. Previous research has repeatedly demonstrated that perceptual fluency is affectively positive, yet this was not found to be the case here. Although some possible explanations were suggested, there is a need to further explicate fluency to try to understand when perceptual or conceptual matter most-if at all. A starting point could simply be re-conceptualizing Bornstein and D'Agostino's (1992, 1994) model as the fluency/attributional model, losing the perceptual component in its name as it fails to consider the contributions of conceptual fluency.

That said, this study did contain a few limitations worth acknowledging as they point to additional needed studies. The first relates to the nature of the participants themselves. The sample was entirely composed of undergraduate students in the Southeastern United States. Although there is no reason to 
believe that the processing effects that were being investigated in this research should be age or location specific, it would still be desirable to have a more diverse sample.

Another potential limitation relates to the artificial nature of certain components of the research design. Everything was designed to look like what participants would encounter in real life; nevertheless, this is not entirely possible, especially in Study 3 when real news websites were being used as stimulus materials. Although the "realness" of the study was strength, especially with the use of known brands, using screenshots created an artificial web environment. It is, of course, unusual to look at a website and not be able to click on any of the links or be able to scroll down the page. Future research would be greatly served by creating environments that are most like what users experience in everyday life.

A second artificial aspect of this research is that the encoding was caused entirely by instructions - that is, participants were specifically instructed to evaluate a brand or logo (studies 1 and 2) or click on an advertisement (study 3). Although this appeared to work well, it is still an artificial manipulation. After all, individuals are never given instructions on how to look at a webpage whenever they are surfing the internet on their own time. Finding ways to manipulate the formal structure of the advertisement in order to induce different types of processing is one major line of future research. In advertising research, the ultimate goal is to establish the most effective ways to persuade the target audience without their knowledge. Although it would be nice to be able to tell the audience how to encode a message, that is never going to happen. Instead, it is possible to try to explore ways to change the features of the message in order to induce the type of processing in which you are interested.

Another important research question that would build on the current results would be to investigate how long the increase in fluency can last. Past research investigating implicit memory performance has suggested that the durability of cognitive primes can last quite a considerable time. In fact, implicit memory measures have shown enhanced performance after an entire week has passed (Tulving, Schacter, \& Stark, 1982). An interesting question, then, is to see how long a prime with a known brand would last. This question is particularly important when thinking about the many messages an individual receives in the course of a day. Future research could explore what Bargh (2006) has termed "second generation" priming questions. If we know that showing the ad for Nike or Adidas primes those brand names, what happens when both are shown to participants? In other words, which "prime" wins? Or would they simply cancel each other out?

The answer to that question could, in part, rely on various moderators that could play an important role in determining the effectiveness of a prime. For instance, familiarity has been used as a moderator in past advertising research (Coates, Butler, \& Berry, 2006). Could brand familiarity moderate the effectiveness of the prime, therein setting up a mediation-moderation model of advertising effectiveness? 
Finally, future endeavors could also use behavioral measures as the key dependent variable. Although it is nice to be able to demonstrate that implicit memory performance correlates with attitudes, an even better outcomeespecially from the perspective of advertising - is to demonstrate that there could be actual changes in behavior. Although the design of those types of experiments can be tricky, behavioral outcomes have been used in consumer behavior research in the past and so trying to determine the relationship between fluency and behavior could be fruitful.

In summary, though, the present research makes a number of significant contributions to the current literature. First, it differentiates between conceptual and perceptual fluency. In that differentiation, results suggest that for known brands, it is conceptual rather than perceptual fluency that drives positive affect - a clear break from most prior research. Second, it demonstrates that an awareness of that fluency may dampen or extinguish any positive gains made by the prior exposure. This suggests that if consumers make effortful attempts to consider their evaluations of brands, they are much less likely to be susceptible to the influence of advertisements.

\section{Bibliography}

Aiken, L. S., \& West, S. G. (1991). Multiple regression: Testing and interpreting interactions. London: Sage Publications.

Bargh, J. A. (2006). What have we been priming all these years? On the development, mechanisms, and ecology of nonconscious social behavior. European Journal of Social Psychology, 36(2), 147-168.

Barnhardt, T. M., \& Geraci, L. (2008). Are awareness questionnaires valid? Investigating the use of posttest questionnaires for assessing awareness in implicit memory tests. Memory \& Cognition, 36(1), 53-64.

Bornstein, R. F., \& D'Agostino, P. R. (1992). Stimulus recognition and the mere exposure effect. Journal of Personality and Social Psychology, 63(4), 545-552.

Bornstein, R. F., \& D'Agostino, P. R. (1994). The attribution and discounting of perceptual fluency: Preliminary tests of a perceptual fluency/attributional model of the mere exposure effect. Social Cognition, 12(2), 103-128.

Bowers, J. S., \& Schacter, D. L. (1990). Implicit memory and test awareness. Journal of Experimental Psychology. Learning, Memory, and Cognition, 16(3), 404-416.

Butler, L. T., \& Berry, D. C. (2004). Understanding the relationship between repetition priming and mere exposure. British Journal of Psychology, 95 (4), 467-487.

Choi, Y. K., Lee, S., \& Li, H. (2013). Audio and Visual Distractions and Implicit Brand Memory: A Study of Video Game Players. Journal of Advertising, 42(2-3), 219-227.

Coates, S. L., Butler, L. T., \& Berry, D. C. (2004). Implicit memory: A prime example for brand consideration and choice. Applied Cognitive Psychology, 18 (9), 1195-1211.

Coates, S. L., Butler, L. T., \& Berry, D. C. (2006). Implicit memory and consumer choice: The mediating role of brand familiarity. Applied Cognitive Psychology, 20 (8), 1101-1116.

Finlay, K., Marmurek, H. H. C., \& Morton, R. (2005). Priming effects in explicit and implicit memory for textual advertisements. Applied Psychology: An International Review, 54 (4), 442-455. 
Hansen, J., \& Wanke, M. (2009). Liking what's familiar: The importance of unconscious familiarity in the mere-exposure effect. Social Cognition, 27 (2), 161-182.

Hervet, G., Guerard, K., Tremblay, S., \& Chtourou, M. S. (2011). Is banner blindness genuine? Eye tracking internet text advertising. Applied Cognitive Psychology, 25(5), 708-716.

Jacoby, L. L., Kelley, C., Brown, J., \& Jasechko, J. (1989). Becoming famous overnight: Limits on the ability to avoid unconscious influences of the past. Journal of Personality and Social Psychology, 56 (3), 326-338.

Jacoby, L. L., \& Kelley, C. M. (1987). Unconscious influences of memory for a prior event. Personality and Social Psychology Bulletin, 13 (3), 314-336.

Krishnan, H. S., \& Shapiro, S. (1996). Comparing implicit and explicit memory for brand names from advertisements. Journal of Experimental Psychology: Applied, 2(2), 147-163.

Lee, A. Y. (2002). Effects of implicit memory on memory-based versus stimulusbased brand choice. Journal of Marketing Research, 39(4), 440-454.

Lee, A. Y., \& Labroo, A. A. (2004). The effect of conceptual and perceptual fluency on brand evaluation. Journal of Marketing Research, 41(2), 151-165.

MacKenzie, S., Lutz, R., \& Belch, G. (1986). The role of attitude toward the ad as a mediator of advertising effectiveness: A test of competing explanations. Journal of Marketing Research, 23(2), 130-143.

Mulligan, N. W. (2004). Memory: Implicit versus explicit. In L. Nadel (Ed.), Encyclopedia of cognitive science (pp. 1114-1120). London: Nature Publishing Group/MacMillan.

Northup, T., \& Mulligan, N. W. (2013a). Conceptual implicit memory in advertising research. Applied Cognitive Psychology, 27(1), 127-136.

Northup, T., \& Mulligan, N. (2013b). Online advertisements and conceptual implicit memory: Advances in theory and methodology. Applied Cognitive Psychology, 28(1), 66-78.

Parker, A., \& Dagnall, N. (2009). Effects of retrieval practice on conceptual explicit and implicit consumer memory. Applied Cognitive Psychology, 23(2), 188-203.

Roediger, H. L., \& McDermott, K. B. (1993). Implicit memory in normal human subjects. In F. Boller \& J. Grafman (Ed.), Handbook of Neuropsychology, vol. 8, 63-131. Amsterdam: Elsevier.

Schacter, D. L. (1987). Implicit memory: History and current status. Journal of Experimental Psychology: Learning, Memory, and Cognition, 13(3), 501-518.

Tulving, E., \& Schacter, D. L. (1990). Priming and human memory systems. Science, 247(4940), 302.

Tulving, E., Schacter, D. L., \& Stark, H. A. (1982). Priming effects in word-fragment completion are independent of recognition memory. Journal of Experimental Psychology: Learning, Memory, and Cognition, 8(4), 336-342.

Vandeberg, L., Murre, J., Voorveld, H., \& Smit, E. (2015) Dissociating explicit and implicit effects of cross-media advertising. International Journal of Advertising, 34(5), 744-764.

Yang, M., \& Roskos-Ewoldsen, D. R. (2007). The effectiveness of brand placements in the movies: levels of placements, explicit and implicit memory, and brandchoice behavior. Journal of Communication, 57(3), 469-489.

Yang, M., Roskos-Ewoldsen, D. R., Dinu, L., \& Arpan, L. M. (2006). The effectiveness of "in-game" advertising: Comparing college students' explicit and implicit memory for brand names. Journal of Advertising, 35 (4), 143-152.

Yoo, C. Y. (2007). Implicit memory measures for web advertising effectiveness. Journalism \& Mass Communication Quarterly, 84(1), 7-23. 
Yoo, C. Y. (2008). Unconscious processing of web advertising: Effects on implicit memory, attitude toward the brand, and consideration set. Journal of Interactive Marketing, 22 (2), 2-18.

Yoo, C. Y. (2010). Effects beyond click-through: Incidental exposure to web advertising. Journal of Marketing Communications, 15(4), 227-246.

Zajonc, R. B. (1968). Attitudinal effects of mere exposure. Journal of Personality and Social Psychology Monographs, 9(2 Pt. 2), 1-27. 
